\title{
UMA TEORIA TERMODINÂMICA PARA BRISAS: TESTE UTILIZANDO SIMULAÇÕES NUMÉRICAS
}

\author{
CLÊNIA RODRIGUES ALCÂNTARA ${ }^{1}$ e ENIO PEREIRA DE SOUZA ${ }^{2}$ \\ ${ }^{1}$ Unidade Acadêmica de Ciências Atmosféricas - Universidade Federal de Campina Grande. \\ Rua Aprígio Veloso, 882, Bodocongó, Bloco CL, CEP 58109-080, Campina Grande, PB. \\ ${ }^{2}$ Instituto de Astronomia Geofísica e Ciências Atmosférica da Universidade de São Paulo (USP). \\ Rua do Matão, 1226, Cidade Universitária, CEP 05508-090, São Paulo, SP. \\ E-mail: clenia@model.iag.usp.br
}

Recebido Abril 2007 - Aceito Junho 2007

\begin{abstract}
RESUMO
O objetivo deste trabalho foi testar uma teoria termodinâmica em brisas marítimas-terrestres acopladas com brisas de vale-montanha através de simulações numéricas tridimensionais em uma região da costa leste do Nordeste Brasileiro, considerando a presença e a ausência da topografia. Embora o contraste de temperatura entre as superfícies seja importante na formação da brisa, a eficiência termodinâmica é fundamental na determinação da sua intensidade. Tem-se que a inclinação faz com que a diferença de pressão entre dois pontos fique maior durante o dia e menor durante a noite contribuindo para a formação de brisas marítimas mais intensas e de brisas terrestres menos intensas, respectivamente. A máxima queda de pressão ocorre por volta de três horas antes da máxima intensidade da brisa. Isso porque grande parte da energia disponibilizada para as circulações é gasta para vencer dissipação, principalmente, no período diurno, quando esses processos são realmente efetivos. Do ponto de vista puramente termodinâmico a inclinação da montanha atua para intensificar a brisa durante o dia e para enfraquecê-la durante a noite.
\end{abstract}

Palavras-chave: Brisa, Teorias Termodinâmicas, Trabalho de Expansão, BRAMS.

\begin{abstract}
A THERMODYNAMIC THEORY FOR BREEZES: TEST USING NUMERIC SIMULATIONS

In this work we test a thermodynamic theory for sea-land breeze coupled with valley-mountain breeze through tri-dimensional numeric simulations. We verify the presence of such a breeze in Northeast Brazil's east coast and perform experiments with and without the topography of the region. Although the temperature contrast between two surfaces is important in forming the breezes, the thermodynamic efficiency is a key parameter for the breeze intensity. The presence of the slope causes the pressure difference between two points to increase during the day and to decrease during the night. This contributes for more intense daytime breeze and less intense nighttime ones. The maximum pressure drop occurs about three hours prior the maximum intensity of the sea breeze. This is because most of the available energy for the circulations is used to overcome dissipation, mainly during daytime. From a purely thermodynamic viewpoint, the slope of a mountain acts to intensifying the daytime breeze and to weaken the nighttime breeze.
\end{abstract}

Keywords: breeze, thermodynamic theory, expansion work, BRAMS.

\section{INTRODUÇÃO}

As circulações do tipo brisa são, basicamente, uma resposta da atmosfera a um gradiente de entropia que se estabelece à superfície (Souza et al., 2000). Ou seja, elas surgem pelas diferenças entre os fluxos de calor sensível e latente existentes entre duas superfícies distintas (Mahfouf et al., 1987; Avissar e Pielke, 1989; Mahrer e Pielke, 1978).
O mecanismo gerador dessas circulações é semelhante, seja ela gerada devido ao contraste terra/mar (brisa marítimaterrestre), inclinações do terreno (brisa de vale-montanha), ou outras heterogeneidades, tais como, variações espaciais na evapotranspiração à superfície e reflexão/absorção de radiação solar (circulações não-clássicas) [Segal e Arritt, 1992]. Para as brisas marítimas, as diferenças nas capacidades caloríficas das superfícies continentais e oceânicas são transmitidas para a 
camada limite pelo fluxo de calor sensível à superfície. As diferenças resultantes na temperatura da camada limite, mais quente durante o dia sobre a terra e durante a noite sobre a água, gera um centro de baixa pressão sobre a superfície mais quente que resulta em movimento ascendente, escoamento da superfície mais fria para a mais quente, escoamento de retorno alguns níveis acima e um ramo descendente sobre a superfície mais fria, definindo uma célula de circulação local em um plano vertical (Silva Dias et al., 2004). À noite, a circulação é invertida, pois o oceano passa a estar mais quente que a superfície continental, caracterizando a brisa terrestre.

Vários estudos observacionais e numéricos têm mostrado a importância destas circulações para as regiões afetadas (Hsu, 1970, Childs e Raman, 2005; Srinivas e Venkatesan, 2005, Lemonsu et al., 2006; Zawar-Reza e Sturman, 2006). As variações locais induzidas por elas têm conseqüências práticas importantes, por exemplo, nas condições de tempo local e na estrutura da camada limite, o que pode ser significante na dispersão de poluentes dos grandes centros urbanos (Mangia et al., 2004). Outra linha de estudos mostra os impactos do escoamento de grande escala na circulação de brisa e o acoplamento de sistemas de meso escala com sistemas de escalas maiores (Estoque, 1962; Chang et al., 1982; Arrit, 1993; Zhong e Talke, 1993; Melas et al., 1998a; Melas et al., 1998b; Mangia et al., 2004).

Souza et al. (2000) formularam uma teoria termodinâmica que permitiu quantificar os efeitos combinados do desmatamento com a inclinação do terreno na intensidade das circulações diretas de meso escala, através do cálculo da diferença de pressão entre dois pontos. Essa teoria baseia-se no princípio da convecção como uma máquina térmica (Rennó e Ingersoll, 1996), que vem sendo aplicada em outros fenômenos atmosféricos, tais como furacões (Emanuel, 1986), tornados e trombas d'água (Rennó e Bluestein, 2001). Recentemente, Adams e Rennó (2005) a utilizaram para estudar a irreversibilidade presente em modelos numéricos através de um modelo global idealizado.

Uma característica de estudos baseados em dados observacionais são os erros associados ao instrumento de medição e de leitura que podem causar um erro médio de 15 a $30 \%$ no resultado final da teoria, dependendo da magnitude do termo em questão (Souza, 2004). Quando se utilizam modelos numéricos para este tipo de aplicação, parte destes erros é eliminada e o conjunto de dados utilizado passa a ser mais confiável (embora simulações numéricas também apresentem alguns erros, tais como, aqueles decorrentes da resolução das equações diferenciais utilizando-se os métodos numéricos). Além disso, por exemplo, dados retirados de um ponto de grade apresentam consistência de acordo com a lei dos gases, o que não é necessariamente obtido com medidas instrumentais.
Por fim, simulações desse tipo permitem um maior detalhamento dessas circulações.

A teoria formulada por Souza et al. (2000) apresenta vários parâmetros cuja determinação, além de estar sujeita ao erro instrumental, dependia de determinações subjetivas, o que é mais uma fonte de incerteza. Para ter uma avaliação mais consistente da teoria, que serviu de base para o cálculo do trabalho de expansão em circulações tipo brisa (Souza, 2004), o presente trabalho tem como objetivo testar a teoria de diferença de pressão formulada por Souza et al. (2000) em brisas marítimas-terrestres acopladas com brisas de vale-montanha utilizando simulações numéricas tri-dimensionais e com topografia realística. A região de estudo será uma área na costa leste do Nordeste Brasileiro. Essa teoria possibilita verificar vários fatores que podem influenciar nas características dessas circulações, tais como, intensidade e profundidade.

\section{DADOS E METODOLOGIA}

\subsection{Teoria termodinâmica aplicada às circulações locais}

O objetivo da teoria desenvolvida por Souza et al. (2000) foi obter uma expressão simples para a diferença de pressão entre dois pontos ao longo do ramo inferior de uma circulação tipo brisa. Definem-se, portanto, os pontos A e B como representativos da região onde uma parcela começa a absorver calor da superfície e da região onde a absorção atinge o máximo, respectivamente. É entre esses dois pontos que a queda de pressão é avaliada. Souza (2004) apresenta uma representação esquemática desses pontos. Como a teoria foi apresentada em detalhes em Souza et al. (2000) e Souza (2004), aqui é apresentada apenas uma breve descrição.

Considere-se a equação da energia para uma parcela de ar em estado estacionário (Haltiner e Martin, 1957),

$$
\mathrm{d}\left(\frac{1}{2}|\overrightarrow{\mathrm{v}}|^{2}+\mathrm{gz}\right)+\operatorname{adp}-\overrightarrow{\mathrm{f}} \cdot \mathrm{d} \vec{\ell}=\mathrm{C}
$$

em que é o vetor velocidade, g é a aceleração da gravidade, $\alpha$ é o volume específico, $\mathrm{p}$ é a pressão, $\overrightarrow{\mathrm{f}}$ é a força de fricção por unidade de massa e $\mathrm{d} \vec{\ell}$ é um incremento de distância ao longo do caminho da parcela de ar. E o primeiro e o segundo princípios da termodinâmica, aplicados ao ar úmido:

$$
T d s=d\left(c_{p} T+L_{v} r\right)-\alpha d p
$$


em que T é a temperatura absoluta do ar, s é a entropia específica do ar, $\mathrm{c}_{\mathrm{p}}$ é o calor específico do ar seco sob pressão constante, $\mathrm{L}_{v}$ é o calor latente específico de vaporização e $\mathrm{r}$ é a razão de mistura do vapor d'água.

De acordo com Rennó et al. (1998), define-se a fração da dissipação total causada por fricção próxima à superfície na forma:

$$
\gamma \equiv \frac{\int_{a}^{b} \vec{f} \cdot d \vec{\ell}}{\int \vec{f} \cdot d \vec{\ell}}
$$

e a eficiência termodinâmica da circulação convectiva como

$$
\eta \equiv \frac{\int T d s}{\int_{a}^{b} T d s}
$$

Integrando as equações (1) e (2) ao longo de uma circulação fechada, e em seguida, ao longo de uma linha de corrente e, por fim, combinando os resultados com as equações (3) e (4), obtém-se:

$$
(1-\gamma \eta) \int_{A}^{B} \alpha d p \approx-\gamma \eta \int_{A}^{B} d\left(c_{p} T+L_{v} r\right)-\int_{A}^{B} g d z
$$

Para melhor se entender a contribuição da inclinação do terreno nos processos estudados, Souza et al. (2000) e Souza (2004) dividiram a diferença de temperatura entre A e B em duas partes, uma adiabática $\left(\Delta \mathrm{T}_{\mathrm{ad}}\right)$ e outra não-adiabática $\left(\Delta \mathrm{T}_{\text {na }}\right)$. Logo,

$$
\Delta T_{n a}=\Delta T+\frac{g}{c_{p}} \Delta z
$$

em que $\Delta \mathrm{T}=\mathrm{T}_{\mathrm{B}}-\mathrm{T}_{\mathrm{A}}$ é a diferença de temperatura e $\Delta \mathrm{Z}=\mathrm{Z}_{\mathrm{B}}-\mathrm{Z}_{\mathrm{A}}$ é a diferença de altitude entre os pontos A e B. Então, a queda de pressão entre os pontos A e B é dada por:

$$
\Delta p \approx p_{A}\left\{1-\exp \left[\frac{\gamma \eta}{(\gamma \eta-1) R}\left(\frac{c_{p} \Delta T_{n a}}{\overline{T_{S}}}+\frac{L_{v} \Delta r}{\overline{T_{S}}}\right)-\frac{\Delta z}{H_{S}}\right]\right\}
$$

em que $\Delta \mathrm{p} \equiv \mathrm{p}_{\mathrm{B}}-\mathrm{p}_{\mathrm{A}}$ é a queda de pressão e $\Delta \mathrm{r}=\mathrm{r}_{\mathrm{B}}-\mathrm{r}_{\mathrm{A}}$ é a variação da razão de mistura entre os pontos $\mathrm{A} e \mathrm{~B}, \overline{\mathrm{T}}_{\mathrm{S}}$ é a média da temperatura do ar próximo à superfície, observada entre os pontos de referência, e $H_{S} \equiv R \bar{T}_{S} / g$ é uma altura de escala. Note-se que, da forma como é definido na Equação (7),

$\Delta \mathrm{p}$ significa a queda de pressão de A para $\mathrm{B}$, de modo que valores positivos significam diminuição da pressão.
O primeiro termo da Equação (7) é o que está diretamente ligado à circulação.Ele é devido à absorção de calor sensível entre os pontos e é chamado aqui de diferença de pressão não-hidrostática. $\mathrm{O}$ segundo termo mostra a absorção de calor latente quando há condensação ao longo da circulação. Por fim, o terceiro termo é a combinação da queda de pressão hidrostática devido à diferença de altura entre os pontos $\mathrm{A}$ e $\mathrm{B}$ e uma expansão não-adiabática ao longo do aclive.

A eficiência termodinâmica $\eta$ é obtida a partir da seguinte equação (Souza et al., 2000):

$$
\eta=\frac{g Z}{c_{p} T_{h}}
$$

em que $T_{h}$ é a temperatura da região onde calor é absorvido (próximo à superfície) e $\mathrm{Z}$ é a profundidade da camada limite turbulenta. Essa formulação foi usada com sucesso por Tian e Parker (2003) em um estudo sobre o papel da orografia no desenvolvimento de convecção rasa.

\subsection{Características do modelo utilizadas nas simulações e descrição dos dados observados}

O modelo utilizado foi o Brazilian Regional Atmospheric Modelling System - BRAMS para a realização das simulações com inicialização heterogênea e análises do Modelo Global do Centro de Previsão de Tempo e Estudos Climáticos (CPTEC), com resolução T126L28.

Para fornecer uma melhor resolução espacial, as simulações foram integradas com duas grades aninhadas: a grade externa centrada em $\left(7^{\circ} \mathrm{S} ; 36^{\circ} \mathrm{W}\right)$, com resolução de $20 \mathrm{~km}$ com 55 x 55 pontos de grade; a grade interna foi centrada em $\left(7^{\circ} \mathrm{S}\right.$; $35^{\circ} \mathrm{W}$ ), resolução horizontal de $5 \mathrm{~km}$, com 142 pontos em x e 74 pontos em y. Esta última pode ser vista na Figura 1. Na vertical, a atmosfera foi dividida em 40 níveis com espessuras diferentes, com $20 \mathrm{~m}$ a partir da superfície e aumentando a uma taxa de 1,2 até um máximo de $1000 \mathrm{~m}$ de resolução. Foram considerados também, nove camadas para o solo, que foram $2,0 \mathrm{~m}, 1,75 \mathrm{~m}$, $1,50 \mathrm{~m}, 1,25 \mathrm{~m}, 1,00 \mathrm{~m}, 0,75 \mathrm{~m}, 0,50 \mathrm{~m}, 0,25 \mathrm{~m}, 0,05 \mathrm{~m}$ e todas com grau de saturação inicial de $20 \%$. O 'nuding' para o limite lateral foi de $1800 \mathrm{~s}$, para o centro do domínio foi de $21600 \mathrm{~s}$ e para o topo do domínio foi de 10800 s. As parametrizações utilizadas foram: Chen e Cotton (1983), para radiação de onda curta e onda longa, o LEAF-3 com modelo solo/vegetação e, para a difusão turbulenta, deformação anisotrópica segundo Smagorinsky (1963). A microfísica (Walko et al. 1995) foi ativada no nível 2 e a parametrização de cúmulos não foi ativada.

Esta última opção foi adotada para evitar que o modelo produzisse chuva irrealistamente. 
Desta forma, teve-se o cuidado de se produzir simulações não-saturadas e, assim, o termo ligado à umidade na Equação (7) pôde ser desprezado. Além disso, o que permite que esse termo de liberação de calor latente seja desprezado é a falta de processos de condensação, principalmente ao longo da circulação.O fato de o teor de umidade ser alto é irrelevante para o termo de absorção de calor latente, desde que não haja mudança de fase que possa alterar a energia da circulação.

Foram realizadas duas simulações com topografia (Topo) e sem topografia (STopo), ambas com início à 00 UTC (horário local é UTC menos 3 horas) do dia 01 de novembro de 2003 . O modelo foi integrado por $288 \mathrm{~h}$ e as saídas foram produzidas a cada hora. Porém, os resultados serão mostrados a partir de 00 UTC de 03 de novembro.

Todos os cálculos e as análises que seguem foram feitos sobre as latitudes de $7^{\circ} \mathrm{S}$ e $8^{\circ} \mathrm{S}$. Após verificar-se o comprimento horizontal das brisas, tomou-se como ponto de referência sobre o continente a longitude de $35,25^{\circ} \mathrm{W}$ (ponto B) e sobre o oceano, de $34,75^{\circ} \mathrm{W}$ (ponto $\mathrm{A}$ ).

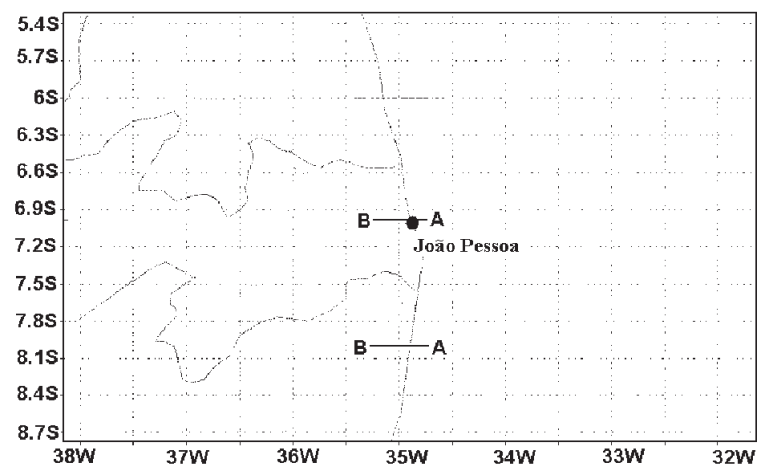

Figura 1 - Área compreendida pela grade interna utilizada nas simulações, com as indicações dos pontos $\mathrm{A}\left(34,75^{\circ} \mathrm{W}\right)$ e $\mathrm{B}\left(35,25^{\circ} \mathrm{W}\right)$ ao longo das latitudes de $7^{\circ} \mathrm{S}$ e $8^{\circ} \mathrm{S}$ e da cidade de João Pessoa - PB.

Assim, a diferença de altitude entre os pontos é de aproximadamente $100 \mathrm{~m}$, ao longo de $7^{\circ} \mathrm{S}$, e $140 \mathrm{~m}$ ao longo de $8^{\circ} \mathrm{S}$, como mostra o perfil da topografia sobre essas latitudes (Figura 2). De forma resumida, os experimentos mostrados neste trabalho são descritos na Tabela 1.

Tabela 1 - Experimentos realizados e suas descrições.

\begin{tabular}{|c||c|}
\hline Experimento & Descrição \\
\hline Topo7S & $\begin{array}{c}\text { Simulação com topografia e resultados } \\
\text { analisados sobre a latitude } 7^{\circ} \mathrm{S}\end{array}$ \\
\hline \hline Topo8S & $\begin{array}{c}\text { Simulação com topografia e resultados } \\
\text { analisados sobre a latitude } 8^{\circ} \mathrm{S}\end{array}$ \\
\hline STopo7S & $\begin{array}{c}\text { Simulação sem topografia e resultados } \\
\text { analisados sobre a latitude } 7^{\circ} \mathrm{S}\end{array}$ \\
\hline
\end{tabular}

Para se calcular a eficiência termodinâmica (Equação 8) nas simulações, Th foi considerada a temperatura do primeiro nível atmosférico disponível (10m acima da superfície). A profundidade da camada limite turbulenta $Z$ é obtida diretamente da saída do modelo a partir do perfil de temperatura potencial. Esse cálculo é mais preciso durante o dia. À noite, quando a turbulência é predominantemente mecânica, a determinação da altura da CLP é menos clara.

Contudo, os baixos valores encontrados durante a noite são coerentes. Th e $\mathrm{Z}$ são obtidas em cada ponto de grade existente entre as longitudes de $35,25^{\circ} \mathrm{W}$ e $34,75^{\circ} \mathrm{W}$. Dessa forma, a eficiência utilizada nos cálculos que seguem é a média das eficiências observadas em cada ponto de grade ao longo de uma latitude e entre as longitudes de $35,25^{\circ} \mathrm{W}$ e $34,75^{\circ} \mathrm{W}$.

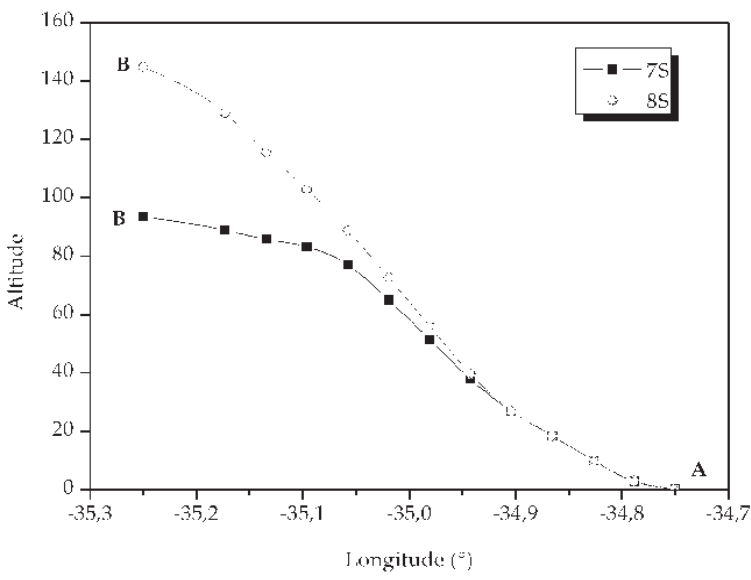

Figura 2 - Perfil de topografia ao longo das latitudes $7^{\circ} \mathrm{S}$ e $8^{\circ} \mathrm{S}$ e entre os pontos $\mathrm{A}\left(34,75^{\circ} \mathrm{W}\right)$ e $\mathrm{B}\left(35,25^{\circ} \mathrm{W}\right)$.

Outro parâmetro-chave para a teoria é a fração de dissipação que ocorre próximo à superfície g. Stull (1988) sugeriu, após fazer simulações de grandes turbilhões, que a maior parte da dissipação observada ao longo da circulação ocorre próxima à superfície. Souza et al. (2000) utilizaram o valor de $\mathrm{g}=1$, o que implica que toda a dissipação supostamente ocorre próxima à superfície e assim, a equação (7) iria prever a máxima intensidade possível das circulações. Para se saber qual o melhor valor a ser utilizado para g, foi testada a sensibilidade da teoria de diferença de pressão [Equação (7)] a esse parâmetro, calculando o erro médio quadrático entre o valor previsto pela teoria e o valor encontrado nas simulações para os experimentos Topo7S, Topo8S e STopo7S, o resultado é mostrado na Figura 3.

Assim, os menores erros apresentam-se entre $g=0,5$ e $\mathrm{g}=0,6$, ou seja, de $50 \%$ a $60 \%$ da dissipação observada ao longo da circulação ocorre próximo a superfície. Tal resultado mostra-se coerente com a consideração de Rennó (2001) para a aplicação dessa teoria à circulação de Hadley no qual considerou $\mathrm{g}=0,5$. 
Neste trabalho, os resultados que serão mostrados baseiam-se em um valor de $\mathrm{g}=0,6$. Esse valor mostrou-se mais adequado para os experimentos realizados.

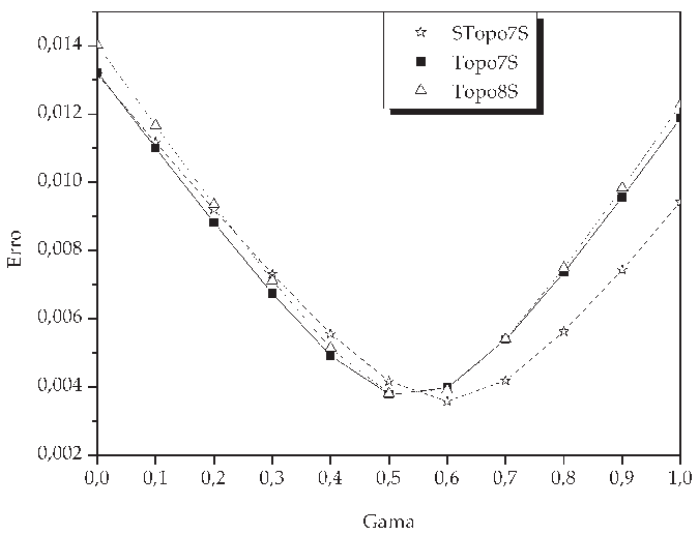

Figura 3 - Erro médio quadrático em função do parâmetro g nos experimentos Topo7S, Topo8S e STopo7S.

Mesmo com bons experimentos numéricos, não é fácil "enxergar" esses sistemas quando estão contidos em umescoamento de grande escala.

A metodologia de Souza et al. (2000) para a visualização destas circulações consiste em tomar certa variável e escrevêla como a soma de uma média no tempo, que representaria a variável em escala sinótica, mais uma perturbação, que representaria as circulações. Assim $\chi(t)=\chi+\chi^{\prime}(t)$.Portanto, o efeito da brisa pode ser obtido de $\chi^{\prime}(\mathrm{t})=\chi(\mathrm{t})-\bar{\chi}$.

Além das simulações, também foram utilizados dados observados de uma localidade litorânea afetada pelas brisas, que se encontra dentro da área compreendida pela grade interna utilizada nas simulações: João Pessoa - PB $\left(7,08^{\circ} \mathrm{S} ; 34,83^{\circ} \mathrm{W}\right)$ (ver a Figura 1). Os dados analisados para essa cidade são de temperatura, intensidade e direção do vento durante o mesmo período considerado para as simulações. Esses dados para João Pessoa foram disponibilizados no site REDEMET (www. redemet.aer.mil.br) com resolução temporal de $1 \mathrm{~h}$ e foram coletados na estação convencional de superfície do Aeroporto Castro Pinto.

\section{RESULTADOS E DISCUSSÃO}

\subsection{Validação das simulações}

Nesta seção comparam-se as simulações realizadas com os dados observados para João Pessoa, descritos anteriormente. $\mathrm{Na}$ Figura 4, observa-se a perturbação da componente zonal do vento ao longo da latitude de $7^{\circ} \mathrm{S}$ entre os pontos A e B. As curvas são: a perturbação da componente zonal do vento calculada na simulação com topografia (Topo7S) (u' simulada) e a perturbação da componente zonal do vento observado para João pessoa (u' observada), entre 00 UTC do dia 3 de novembro e 00 UTC do dia 13 de novembro de 2003.

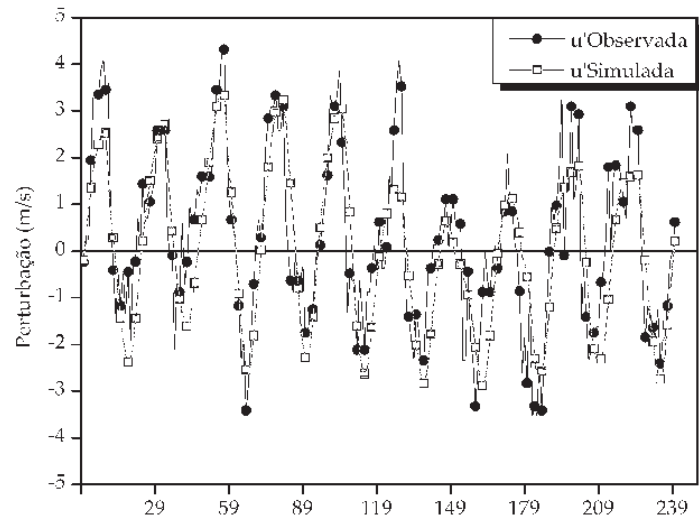

Figura 4 - Perturbação da componente zonal do vento observado em João Pessoa $\left(7,08^{\circ} \mathrm{S} ; 34,83^{\circ} \mathrm{W}\right)$ e média da perturbação da componente zonal do vento simulado ao longo de $7^{\circ} \mathrm{S}$ entre os Pontos $\mathrm{A}\left(34,75^{\circ} \mathrm{W}\right)$ e B $\left(35,75^{\circ} \mathrm{W}\right)$.

Pode-se ver que o modelo é capaz de simular com razoável precisão o ciclo diurno da perturbação do vento tanto em termos de fase quanto de amplitude. Note-se que há uma variação entre os dias e que a simulação acompanha de perto os valores observacionais, com exceção ao dia 08 de novembro, quando o modelo subestima a intensidade da brisa terrestre.



Figura 5 - Temperatura observada em João Pessoa $\left(7,08^{\circ} \mathrm{S} ; 34,83^{\circ} \mathrm{W}\right)$ e média da temperatura simulada ao longo da latitude de $7^{\circ} \mathrm{S}$ entre $35^{\circ} \mathrm{W}$ e o ponto $\mathrm{B}\left(35,25^{\circ} \mathrm{W}\right)$.

Já a Figura 5 mostra a média da temperatura simulada em cada ponto de grade entre os pontos $\mathrm{B}\left(35,25^{\circ} \mathrm{W}\right)$ e $35^{\circ} \mathrm{W}$, que é exatamente no limite da costa, e a temperatura dos dados observados em João Pessoa para o mesmo período. Observa-se que a temperatura nessa localidade varia entre $295,5 \mathrm{~K}\left(22^{\circ} \mathrm{C}\right)$ e $304,5 \mathrm{~K}\left(31^{\circ} \mathrm{C}\right)$. Porém, sem grandes diferenças entre os dias analisados, a não ser pelo período entre a tarde de $08 \mathrm{de}$ 
novembro e a manhã de 09 de novembro, no qual se tem que a temperatura mínima foi maior que nos demais dias e que a temperatura máxima foi menor comparada aos outros dias. Notese que o modelo tende a superestimar as temperaturas mínimas. Isso porque, provavelmente, ele se apresenta mais úmido, pela falta de um processo eficiente de remoção e não represente apropriadamente o resfriamento radiativo noturno.

Percebe-se que a simulação seguiu a tendência dos dados observados, especialmente para a perturbação da componente zonal do vento, na qual se observa uma maior variabilidade entre os dias mostrados. Assim, pode-se considerar que a simulação está em boa concordância com os dados observados para João Pessoa, como pode ser confirmado pelas informações estatísticas mostradas na Tabela 2, em que se observam os coeficientes de correlação (r), raiz do erro médio quadrático (RMSE), RMSE depois de removido um bias constante [(RMSE)BIAS] e os devios-padrão dos dados observados (sOBS) e dos dados simulados (s). Estes últimos parâmetros foram calculados segundo Pielke (2002). Apenas os dados de temperatura apresentam um maior desvio-padrão dos dados observados, em relação aos do modelo. Isso se deve a superestimativa das temperaturas mínimas, já discutida anteriormente.

Tabela 2 - Parâmetros estatísticos para análise do ajuste entre os dados, em que r é o coeficiente de correlação, RMSE é a raiz do erro médio quadrático, (RMSE)BIAS é RMSE retirado um bias constante, sOBS/s são os desvios-padrão dos dados observados e das simulações, respectivamente.

\begin{tabular}{|c||c|c|c|c|}
\hline Variáveis Comparadas & $\mathrm{r}$ & RMSE & (RMSE)BIAS & бовs $/ \sigma$ \\
\hline \hline $\mathrm{u}^{\prime}$ observada $\mathrm{X} \mathrm{u}^{\prime}$ simulada & 0,82 & 1,10 & 1,07 & $1,81 / 1,71$ \\
\hline \hline $\begin{array}{c}\text { Temperatura observada } \mathrm{X} \\
\text { simulada }\end{array}$ & 0,93 & 1,04 & 1,07 & $2,56 / 1,98$ \\
\hline \hline$\Delta \mathrm{P}$ teórica (Topo7S) $\mathrm{X}$ simulada & 0,95 & 0,15 & 0,19 & $0,40 / 0,38$ \\
\hline \hline$\Delta \mathrm{P}$ teórica (Topo8S) $\mathrm{X}$ simulada & 0,95 & 0,15 & 0,18 & $0,39 / 0,36$ \\
\hline \hline$\Delta \mathrm{P}$ teórica (STopo7S) $\mathrm{X}$ simulada & 0,95 & 0,12 & 0,31 & $0,38 / 0,39$ \\
\hline
\end{tabular}

Para as correlações (r) mostradas neste trabalho foi aplicado um teste de significância através da distribuição $\mathrm{t}$ de Student (Bussab e Morettin, 1987). Assim, para o nível de significância de $1 \%$, as correlações são estatisticamente significativas se maiores que 0,16 .

\subsection{Teoria para diferença de pressão entre dois pontos}

Nesta seção comparam-se as simulações realizadas com e sem topografia com a teoria de diferença de pressão (Equação 7).

$\mathrm{Na}$ Figura 6, tem-se a diferença de temperatura entre os pontos B e A, ou seja, entre o continente e o oceano, para Topo $7 \mathrm{~S}$, Topo8S e STopo7S. Logo, valores negativos correspondem ao período noturno quando o oceano está mais aquecido. De forma inversa, valores positivos de diferença de temperatura indicam que o continente está mais aquecido que o oceano e, por sua vez, correspondem à situação de brisa marítima.

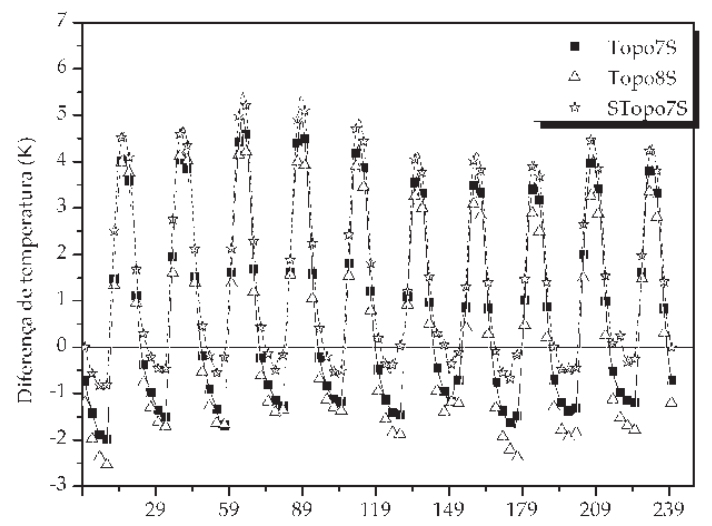

Figura 6 - Diferença de temperatura entre os pontos B $\left(35,25^{\circ} \mathrm{W}\right)$ e A $\left(34,75^{\circ} \mathrm{W}\right)$ para os experimentos Topo $7 \mathrm{~S}$, Topo8S e STopo $7 \mathrm{~S}$.

Percebe-se que a diferença de temperatura para Topo7S mostra-se maior para os casos de brisa marítima do que para os casos de brisa terrestre, atingindo $5 \mathrm{~K}$ e quase $-2 \mathrm{~K}$, respectivamente. No período mostrado, não se observa muita variação dessa diferença. De acordo com esses resultados, seria esperado que as brisas marítimas fossem bem mais intensas que as brisas terrestres, pois a diferença de temperatura entre os módulos dos picos chega a ser de até $3 \mathrm{~K}$. Porém, o que se observa tanto nas simulações (Figura 6), quanto nos dados observados (Figura 5) é que nem sempre isso acontece. O que reforça a influência de outros fatores para a intensidade das circulações de brisa.

Ainda na Figura 6 pode-se observar o efeito da elevação do terreno na diferença de temperatura comparando-se os resultados dos três experimentos. Para o período diurno, em média, ela foi maior no experimento STopo $7 \mathrm{~S}$ do que no Topo7S, e menor no experimento Topo8S. Isso se explica pelo fato de que quanto maior for a altitude menor é a temperatura, em relação ao nível médio do mar. Note-se que a temperatura sobre o mar tende a variar pouco entre os experimentos. Durante a noite, o efeito do resfriamento sobre a montanha se intensifica e a diferença de temperatura chega a ser bem maior, em módulo, no experimento Topo8S.

A Figura 7 traz as perturbações da componente zonal do vento definidas como, ou seja, uma média no tempo menos a variável em cada ponto de grade. Além disso, também foi feita uma média entre os pontos A e B ao longo de cada latitude em estudo. Percebe-se que entre os experimentos Topo7S e Topo8S a diferença nas intensidades dos máximos de velocidade foi muito pequena, ou seja, mesmo aumentando em $40 \mathrm{~m}$ a diferença de altitude entre os pontos A e B, o aumento na intensidade das circulações foi mínimo. 


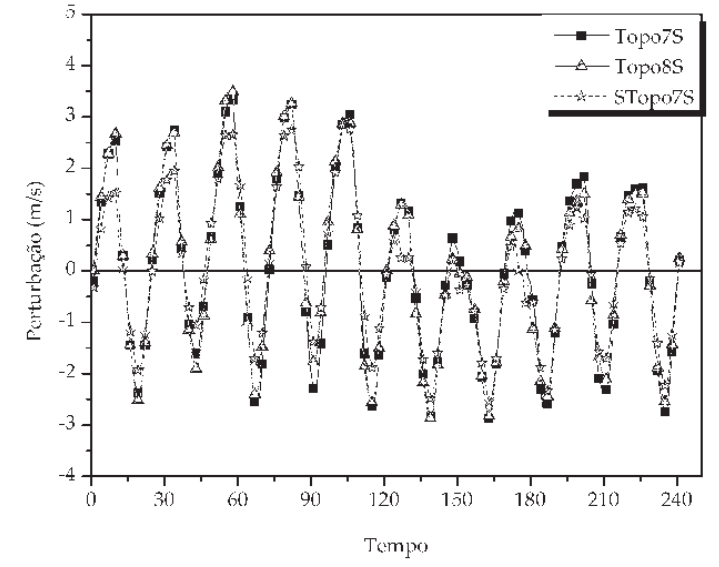

Figura 7 - Perturbação da componente zonal do vento para Topo7S, Topo8S e STopo7S.

Já para o experimento STopo7S, as intensidades das brisas mostraram-se reduzidas, mesmo com a diferença de temperatura mostrando-se maior que nos experimentos com topografia. Isso, mais uma vez, ressalta que o papel da diferença de temperatura no processo não é único. Além disso, o fato de que as velocidades das circulações são maiores nos experimentos Topo7S e Topo8S do que em Stopo $7 \mathrm{~S}$ é devido à expansão do ar que ocorre quando uma parcela tem sua trajetória montanha acima, fazendo com que haja uma absorção extra de entropia, o que fornece mais combustível para a circulação, que se torna mais intensa.

Com efeito, uma das variáveis mais importantes, da qual depende a circulação, é a eficiência termodinâmica. A Equação (7) mostra que a intensidade da circulação depende diretamente dessa variável. Realmente, maiores valores de eficiência implicam em maiores quantidades de energia disponível para as circulações.

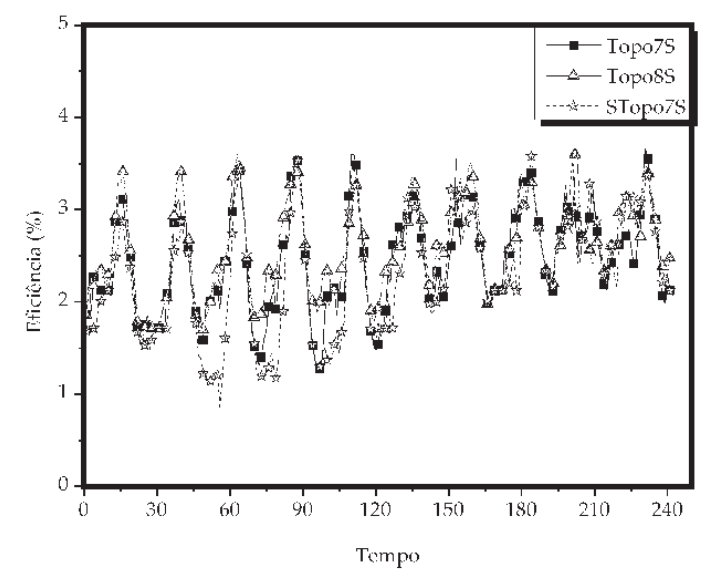

Figura 8 - Eficiência termodinâmica para os experimentos Topo $7 \mathrm{~S}$, Topo8S e STopo7S.
Na Figura 8, vê-se que o valor da eficiência tem forte ciclo diurno e que também varia ao longo do período simulado. Seus maiores valores são encontrados no período diurno, ou seja, poucas horas antes de ocorrer o máximo da intensidade da brisa marítima. Esses máximos foram menores para o experimento STopo7S. Inclusive foram também menores os mínimos, que ocorrem à noite. De forma inversa, o experimento Topo8 $\mathrm{S}$ obteve as maiores eficiências. Isso está associado a uma maior profundidade da camada turbulenta em Topo8S [ver a Equação (8)].Os maiores valores na eficiência de Topo8S indicam que houve mais conversão de energia do calor injetado na circulação a fim de compensar o contraste menor de temperatura, e assim, obter circulações com intensidades próximas àquelas observadas em Topo7S. Para STopo7S, a menor conversão de calor em energia disponível para o sistema pode ter produzido perturbações menores do que as de Topo7S.

A Figura 9 mostra a diferença de pressão observada entre A e B obtida das simulações e calculada através da teoria para Topo7S.

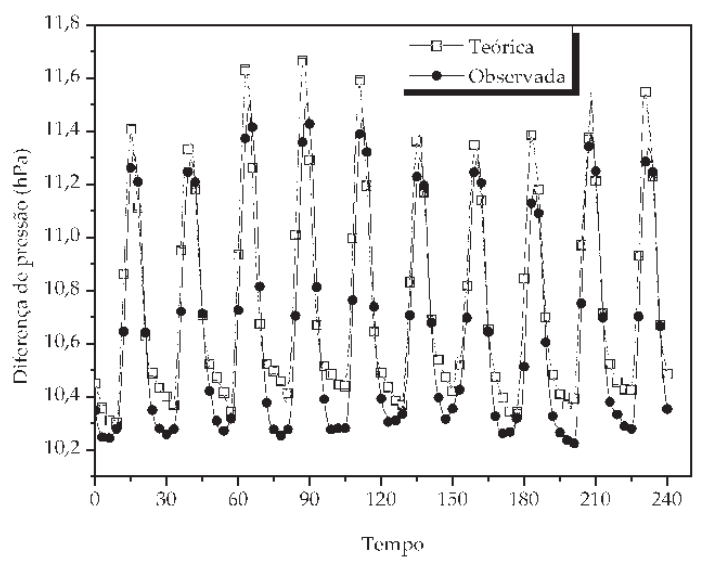

Figura 9 - Diferença de pressão teórica e diferença de pressão simulada para Topo $7 \mathrm{~S}$.

Observa-se que o ajuste entre as curvas teórica e simulada é bastante satisfatório, o que também foi obtido para todos os experimentos (não mostrado aqui). Quando se comparam tais resultados, observa-se que a teoria consegue prever com muito boa aproximação os resultados simulados. E isso pode ser comprovado observando-se a Tabela 2, que traz os valores dos parâmetros estatísticos calculados para a verificação do ajuste existente entre os conjuntos de dados.

Já a Figura 10 traz as diferenças de pressão simuladas para Topo7S, Topo8S e STopo7S. No experimento Topo7S, a diferença de pressão simulada ficou entre 10,2 hPa e 11,5 hPa. Para Topo8S, essas diferenças apresentaram valores maiores devido ao aumento de altitude e ficaram entre $16,0 \mathrm{hPa}$ e 17,3 $\mathrm{hPa}$, aproximadamente. A maior parte dessa queda de pressão é devida à contribuição hidrostática em virtude da diferença de altitude entre os pontos A e B. Por fim, tem-se que para STopo7S, 
a diferença manteve-se entre -0,2 hPa e 1,17 hPa. Assim, percebe-se que quando a circulação não está sob o efeito da elevação essa diferença de pressão torna-se muito pequena.

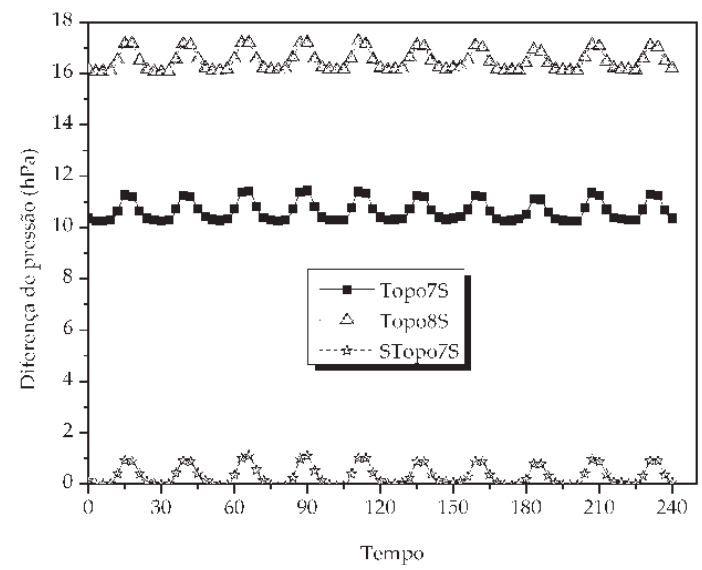

Figura 10 - Diferença de pressão simulada para Topo7S, Topo8S e STopo7S.

Na realidade, quando se anula a contribuição da diferença de altitude o único efeito que resta é devido ao contraste terra/ mar, que possibilita a existência da circulação. Percebe-se também que a diferença de pressão, capaz de forçar a brisa terrestre, é muito pequena. Isso mostra que o estabelecimento da brisa terrestre é mais difícil.

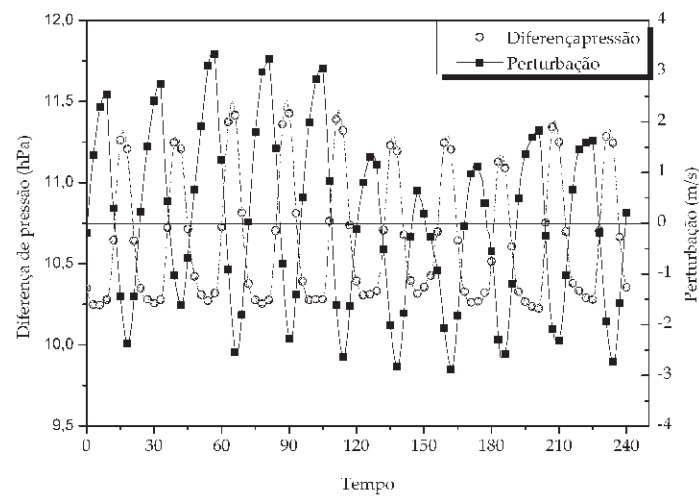

Figura 11 - Diferença de pressão simulada e perturbação simulada da componente zonal do vento para Topo7S.

A Figura 11 traz uma comparação entre a diferença de pressão total simulada e a perturbação simulada da componente zonal do vento e a Figura 12 traz uma comparação entre esta e o fluxo de calor sensível (h) para Topo7S. Os máximos dessa diferença de pressão estão associados aos máximos das perturbações para a brisa marítima e os mínimos estão relacionados com a brisa terrestre, porém ambos com uma pequena defasagem em torno de duas horas para Topo7S, três horas para Topo8S e de duas a quatro horas para STopo $7 \mathrm{~S}$.

Assim, a queda máxima de pressão ocorre antes de se observar a máxima intensidade do vento e que no horário que

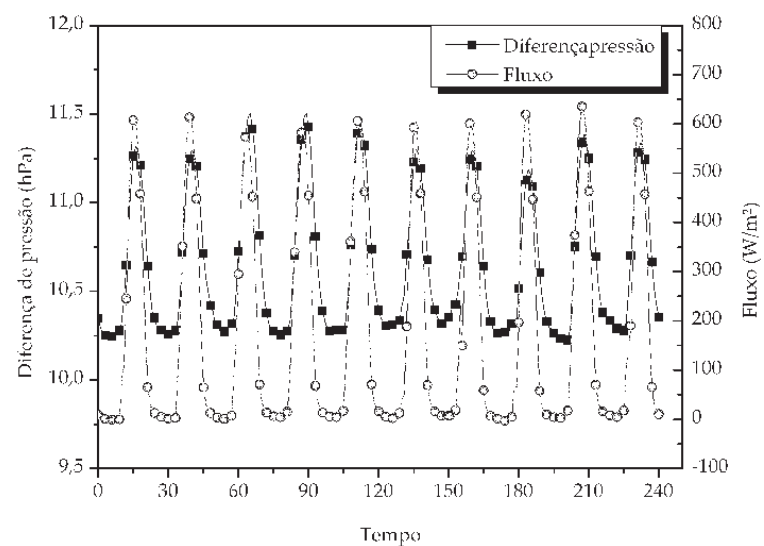

Figura 12 - Diferença de pressão simulada e fluxo de calor sensível (Fluxo) para Topo7S.

se observa essa diferença máxima de pressão também se observa o máximo do fluxo de calor sensível. Isso indica que a turbulência também é máxima no instante da máxima queda de pressão. Assim, muito da energia que é convertida e disponibilizada para a circulação é utilizada para vencer a dissipação imposta pelos processos turbulentos. Por isso, a intensidade máxima da circulação tende a ser observada em um período no qual a quantidade de energia disponibilizada é menor, mas que é utilizada para vencer menos dissipação. Isso é muito importante já que o que se espera é uma correspondência entre a máxima queda de pressão e a máxima intensidade da brisa.

Para comparar os efeitos do contraste terra/mar e vale/montanha nos experimentos realizados, as Figuras 13 e 14 mostram a diferença de pressão não hidrostática para os três experimentos, ou seja, o primeiro termo da Equação 7, porém, com e sem o efeito da topografia para Topo $7 \mathrm{~S}$ e Topo8S, respectivamente. Logo, a pressão não-hidrostática foi calculada utilizando $\Delta \mathrm{T}_{\mathrm{na}}$ nos resultados mostrados na Figura $13 \mathrm{e}$, apenas, $\Delta \mathrm{T}$ na Figura 14

Percebe-se que para os três experimentos, na Figura 13 , esses valores mostram-se muito próximos, principalmente nos casos de brisa terrestre, embora com valores ligeiramente maiores, em módulo, para o experimento Topo8S. Para os casos de brisa marítima, há pequenas diferenças que mostram que nos experimentos Topo7S e Topo8S a diferença de pressão não-hidrostática é maior. Isso ressalta a importância do calor extra, absorvido ao longo do aclive na diminuição da pressão não-hidrostática. Segundo a Equação (6), esse efeito é devido ao fluxo extra que é causado pelo resfriamento adiabático do ar à medida que se desloca para maiores altitudes.

Para se entender melhor esse efeito, a Figura 14 apresenta o mesmo cálculo, porém é usada apenas a diferença de temperatura $\Delta \mathrm{T}$, e não $\Delta \mathrm{T}_{\mathrm{na}}$, na Equação 7. Isso equivale a remover o efeito da montanha na Equação 7. 


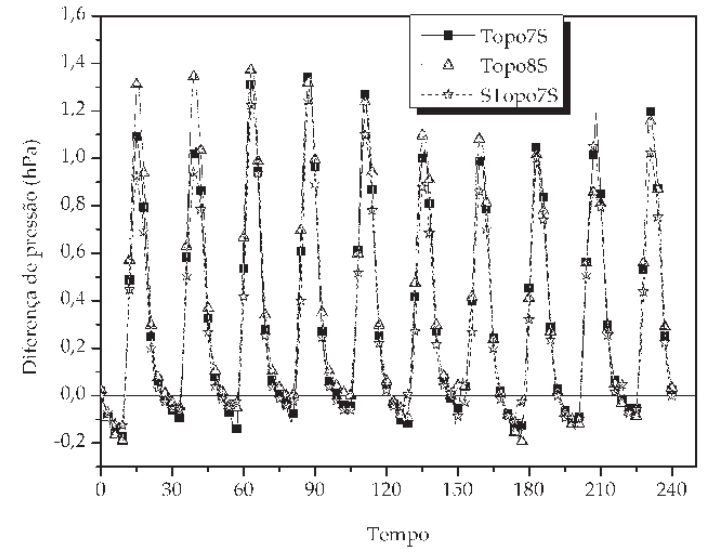

Figura 13 - Diferença de pressão não hidrostática calculada com que inclui o efeito da topografia entre os pontos A e B para Topo $7 \mathrm{~S}$, Topo8S e STopo7S.

No caso da queda de pressão não-hidrostática sem a contribuição da topografia, os valores de Topo8S mostramse maiores (em módulo) do que STopo7S, durante a noite, e menores, durante o dia.

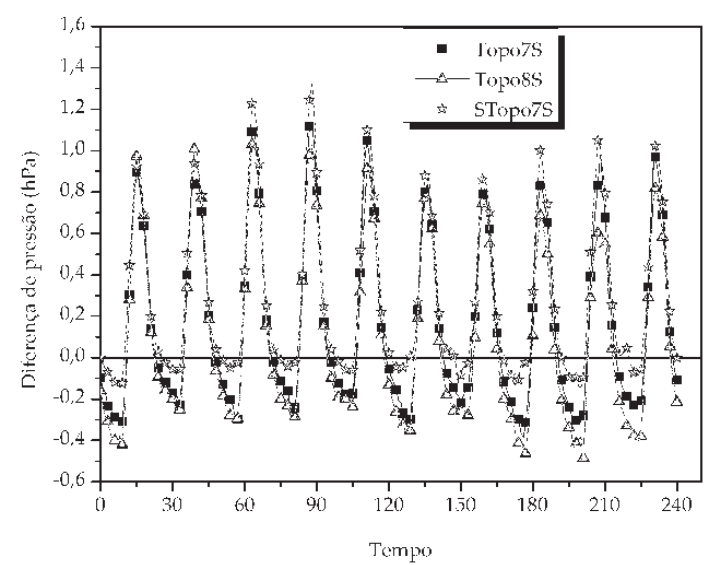

Figura 14 - Diferença de pressão não hidrostática calculada com DT sem considerar o efeito da topografia entre os pontos A e B para Topo7S, Topo8S e STopo7S.

Como a diferença é calculada entre os pontos B e A, valores negativos significam que a pressão em $B$ é relativamente menor em Topo8S do que em STopo7S. Do ponto de vista termodinâmico, esse resultado implica que a combinação de brisa vale/montanha com brisa terra/mar tende a enfraquecer a circulação noturna. Isso acontece porque o ar comprime-se (e se aquece) adiabaticamente, à medida que desce a montanha $\mathrm{e}$ isso incrementa o fluxo de calor para o solo (veja que na Figura 12 , o fluxo de calor sensível é ligeiramente negativo à noite) o que tira energia da circulação. De forma geral, pode-se perceber que a presença da inclinação faz com que a diferença de pressão entre a terra e o mar fique maior durante o dia e menor durante a noite. Esse efeito, isoladamente, contribui para a formação de brisas marítimas mais intensas e de brisas terrestres menos intensas.

\section{CONCLUSÕES}

Foram apresentadas neste trabalho aplicações da teoria termodinâmica de diferença de pressão formulada por Souza et al. (2000), que permite quantificar e caracterizar os efeitos combinados do contraste terra-mar com a inclinação do terreno na intensidade das circulações de brisa. O contraste de temperatura entre as superfícies é determinante na formação das circulações locais, porém outros fatores podem interferir nessa relação, influindo na intensidade das circulações. A eficiência termodinâmica, por exemplo, parece ter um papel fundamental no ciclo diurno dessas circulações e também na comparação entre os experimentos.

Quanto à diferença de pressão, tem-se que a inclinação faz com que esta diferença entre o continente e o mar fique maior durante o dia e menor durante a noite. Esse efeito, isoladamente, contribui para a formação de brisas marítimas mais intensas e de brisas terrestres menos intensas.

Muito da energia disponibilizada para as circulações é gasta para vencer os processos dissipativos. Isso ocorre, principalmente, no período diurno, quando esses processos são realmente efetivos. Isso explica a defasagem encontrada entre a máxima diferença de pressão total e o máximo nas intensidades das circulações. De modo geral, a queda de pressão que é realmente definitiva para a intensidade das circulações (isto é: a parte não hidrostática) é razoavelmente similar entre circulações relacionadas com diferentes topografias. Entretanto, outros fatores, como a eficiência termodinâmica, são mais sensíveis à presença da montanha e interferem na intensidade das circulações. Um aspecto interessante da aplicação da teoria é o fato de a presença da montanha ter um efeito de limitar a intensidade de eventuais circulações noturnas. Isso porque uma parcela de ar deslocando-se montanha abaixo tende a aquecer-se adiabaticamente, mas também tende a perder calor para o solo através do fluxo negativo noturno. Isso então contribui para que a diferença de pressão entre a montanha/terra e o vale/mar seja relativamente menor.

Neste trabalho, a teoria foi testada em casos de montanhas relativamente baixas. Seria interessante estudar o desempenho da teoria em casos de montanhas mais inclinadas. Contudo, o trato da teoria não é trivial uma vez que a presença de montanhas mais acentuadas pode levar as parcelas à saturação e o termo de calor latente na Equação 7 não pode mais ser desprezado. $\mathrm{O}$ trato da precipitação, nessas circulações torna o problema mais complicado. Um estudo nessa linha faz parte da continuação da pesquisa. 


\section{AGRADECIMENTO}

CRA agradece ao CNPq pela bolsa de mestrado que possibilitou o desenvolvimento deste trabalho.

\section{REFERÊNCIAS BIBLIOGRÁFICAS}

ADAMS, D. K.; RENNÓ, N. O. Thermodynamic efficiencies of an idealized global climate model. Clim. Dynamics, v. 25 , p.801-813, 2005.

ARRIT, R. W. Effects of the large-scale flow on characteristic features of the sea breeze. J. Appl. Meteor., v.32, p.116$125,1993$.

AVISSAR, R.; PIELKE, R. A. A parameterization of heterogeneous land surface for atmospheric numerical models and its impact on regional meteorology. Mon. Wea. Rev., v. 117, p. 2113-2136, 1989.

BUSSAB, W. O.; MORETTIN, P. A. Estatística Básica, 4ed. Atual Editora, 1987. p. 288 - 291.

CHANG, L. P.; TALKE, E. S.; SANI, R. L. Development of a two-dimensional finite-element PBL model and two preliminary model applications. Mon. Wea. Rev., v.110, p.2025-2037, 1982.

CHEN, C.; COTTON, W. R. A one-dimensional simulation of the stratocumulus capped mixed layer. Bound.-Layer. Meteor., v.25, p. 289-321, 1983.

CHILDS, P.; RAMAN S. Observations and Numerical Simulations of Urban Heat Island and Sea Breeze Circulations over New York City. Pure appl. geophys. v. 162, p. 1955-1980, 2005.

EMANUEL, K. A. An air-sea interaction theory for tropical cyclones. Part I: steady-state. J. Atmos. Sci. v.43, p. 585604, 1986.

ESTOQUE, M. A. The sea breeze as a function of the prevailing synoptic situation. J. Atmos. Sci., v.19, p.244-250, 1962.

HALTINER, G. J.; MARTIN, F. L. Dynamical and physical meteorology. McGraw-Hill, 1957. 470p.

HSU, S. Coastal air-circulation system: observations and empirical model. Mon. Wea. Rev., v. 98, nº 7, p. 487-509, 1970.

LEMONSU, A.; BASTIN, S.; MASSON, V.; DROBINSKI, P. Vertical structure of the urban boundary layer over Marseille under sea-breeze conditions. Bound.-Layer Meteor., v. 118, p. 477-501, 2006.

MAHFOUF, J.; RICHARD, E.; MASCART, P. The influence of soil and vegetation on the development of mesoscale circulations. J. Clim. Appl. Meteor., v. 26, p. 1483-1496, 1987.

MAHRER, Y.; PIELKE, R. A. The meteorological effect of the changes in surface albedo and moisture. Isr. Meterol. Res. Pap., v.2, p.55-70, 1978.
MANGIA, C.; MARTANO P.; MIGLIETTA, M. M.; MORABITO, A.; TANZARELLA, A. Modelling local winds over the Salento peninsula. Meteorol. Appl., v. 11, p.231-244, 2004.

MELAS, D.; ZIOMAS, I.; KLEMS, O.; ZEREFOR. C. S. Flow dynamics in Athens área under moderate large-scale winds. Atmos. Environ., v. 32, no 12, p. 2209-2222, 1998a.

MELAS, D.; ZIOMAS, I.; KLEMS, O.; ZEREFOR. C. S. Anatomy of sea-breeze circulation in Athens area under weak large-scale ambient winds. Atmos. Environ., v. 32, no 12, p. 2223-2237, 1998 b.

PIELKE, R. A. Mesoscale Meteorological Modeling. 2a ed., Academic Press, 2002. p. 462 - 468.

REDEMET - Rede de meteorologia do comando da aeronáutica - www.redemet.aer.mil.br .

RENNÓ, N. O. Comments on Frictional dissipation in a precipitating atmosphere. J. Atmos. Sci., v.59, p.1173-1177, 2001.

RENNÓ, N. O.; BLUESTEIN, H. B. A simple theory for waterspouts. J. Atmos. Sci. v.58, p.927-932, 2001.

RENNÓ, N. O.; BURKETT, M. L.; LARKIN, M. P. A simple theory for dust devils. J. Atmos. Sci., v.55, p.32244-3252, 1998.

RENNÓ, N. O.; INGERSOLL, A. P. Natural convection as a heat engine: A theory for CAPE. J. Atmos. Sci., v.53, p.572-585, 1996.

SEGAL, M.; ARRIT, R. W. Nonclassical mesoscale circulations caused by surface sensible heat-flux gradients. Bull. Am. Met. Soc., v.73, p.1593 - 1604, 1992.

SILVA DIAS, M. A. F.; SILVA DIAS, P. L.; LONGO, M.; FITZJARRALD, D. R.; DENNING, A. S. River breeze circulation in eastern Amazônia: observation and modelling results. Theor. Appl. Climatol., v. 78, no 1-3, p.111-121, 2004.

SMAGORINSKY, J. General circulation experiments with the primitive equations: I. The basic experiment. Mon. Wea. Rev., v. 91, p. 99-164, 1963.

SOUZA, E. P. Trabalho de expansão e de compressão associado às circulações convectivas. Rev. Bras. Meteoro., v. 19, n. 2, p. 141-148, 2004.

SOUZA, E. P.; RENNÓ, N. O.; SILVA DIAS, M. A. F. Convective circulations induced by surface heterogeneities. J. Atmos. Sci., v.57, p.2915-2922, 2000.

SRINIVAS, C.V.; VENKATESAN, R. A simulation study of dispersion of air borne radionuclides from a nuclear power plant under a hypothetical accidental scenario at a tropical coastal site. Atmos. Environ., v. 39, nº 8, p.1497-1511, 2005.

STULL, R. B. An introduction to boundary layer meteorology. Klerwer Academic Publishers, 1988. 666p. 
TIAN, W.; PARKER, D. J. A modeling study and scaling analysis of orographic effects on boundary layer shallow convection. J. Atmos. Sci., v. 60, p. 1981-1991, 2003.

WALKO, R.; COTTON, W.R.; MEYERS, M.P.; HARRINGTON, J.Y.; New RAMS cloud microphysics parameterization. Part I: The single-moment scheme. Atmos. Res., v. 38, p. 29-62, 1995.
ZAWAR-REZA, P.; STURMAN, A. P. Two-dimensional numerical analysis of a thermally generated mesoscale wind system observed in the Mackenzie Basin, New Zealand. Aust. Met. Mag., v. 55, p. 19-34, 2006.

ZHONG, S.; TALKE, E. S. The effects of large-scale winds on the sea-land-breeze circulations in an area of complex coastal heating. Appl. Meteor., v. 32, p. 1181-1195, 1993. 